Participants will be flagged for notification of all cancer and death registrations, and hospital episode statistics for particular health conditions. More detailed pesticide exposure data will be collected annually, beginning in December 2013.

Results The baseline data will be analysed to provide summary data describing the characteristics of the new cohort. Descriptive statistics will include basic demographics, regional distribution, and summaries of pesticide exposures and lifestyle factors. Response rates, the effect of reminders, the proportion responding online, and factors associated with online response will be presented.

Conclusions This cohort study will play an important role in the post-marketing surveillance of ill-health among professional pesticide applicators in Britain. It will also provide a resource for more detailed investigation of specific health outcomes and exposures.

\section{ASSOCIATION BETWEEN OCCUPATIONAL EXPOSURE TO WOOD DUST AND RISK OF NASOPHARYNGEAL CANCER: A CASE-CONTROL STUDY FROM THAILAND}

${ }^{1} S$ S Sangrajrang, ${ }^{2}$ Ekburanawat, ${ }^{3}$ Ekpanyasakul, ${ }^{4}$ Thetkathek, ${ }^{5}$ Saejew, ${ }^{6}$ Ruangsuwan, ${ }^{7} \mathrm{~T}$ Mannetje. 'National Cancer Institute, Bangkok, Thailand; ${ }^{2}$ Nopparat Rajthanee Hospital, Bangkok, Thailand; ${ }^{3}$ Srinakarinwirot University, Bangkok, Thailand; ${ }^{4}$ Burapa University, Chonburi, Thailand; ${ }^{5}$ Walailak University, Nakhon Si Thammarat, Thailand; ${ }^{6}$ South-East Asia University, Bangkok, Thailand; ${ }^{7}$ Massey University, Wellington, New Zealand

\subsection{6/oemed-2013-101717.391}

Objectives To explore possible association between occupational wood dust exposure and risk of nasopharyngeal cancer (NPC), a matched case - control study was conducted in Bangkok and 6 regional cancer treatment centers in Thailand.

Methods Three hundred and twenty-seven diagnosed NPC cases were compared with 327 age and gender matched controls. Data of socio-demographic characteristics and potential risk factors were collected by personal interviews. Wood dust exposures were assessed by 3 industrial hygienists by reading lifetime occupational histories of the participants with unknown for case-control status. Assessments were done for probability, frequency and intensity of exposure to wood dust. Multivariate analyses were performed adjusting for educational level, smoking status and histories of chronic sinusitis.

Results We found the association between occupational wood dust exposure and NPC risk (OR 1.66, 95\% CI 1.03 - 2.67) especially for those who have definite probability of exposure (OR 1.77, 95\% CI 1.04 - 3.00), moderate frequency of exposure (OR 2.8, 95\% CI $1.22-6.39$ ) and low intensity of exposure (OR 2.29, 95\% CI 1.15 - 4.59).

Conclusion Results of this study shows that occupational exposure to wood dust are likely to be associated with increasing risk of NPC.

\section{AGRICULTURAL ACTIVITIES AND LUNG CANCER MORTALITY IN FRANCE: A NESTED CASE-CONTROL ANALYSIS FROM THE AGRICAN COHORT}

${ }^{1}$ Tual, ${ }^{1} \mathrm{~B}$ Clin, ' ${ }^{2}$ Levêque-Morlais, ${ }^{2}$ Baldi, ' 'Lebailly. 'INSERM, UMR1086-Cancers et Préventions, Caen, France; '2INSERM, ISPED, Centre INSERM U897-EpidemiologieBiostatistique, Bordeaux, France

\subsection{6/oemed-2013-101717.392}

Objectives Farmers have lower lung cancer rates than the general population, due to low smoking rates. Exposure to endotoxins may also play a role. These exposures do not however preclude the role of lung carcinogen exposures in farming activities. We assessed the associations between farming activities and lung cancer from a case-control analysis nested in the French agricultural cohort AGRICAN $(\mathrm{n}=180,060)$.

Methods Incident lung cancer deaths from enrolment (2005$2007)$ to $31 / 12 / 2009(\mathrm{n}=399)$ were individually matched with 4 controls by year of birth and sex by incidence density sampling method. Associations between self-reported lifetime farming activities and lung cancer were estimated using conditional logistic regression, controlling for cigarettes pack years.

Results Lifetime exposure to animals was significantly related to a decreased lung cancer risk (OR $=0.59,95 \%$ CI:0.41-0.84) with a similar association among non-smokers (OR $=0.57,95 \%$ CI: 0.29-1.12). Non-significant decreased risks were observed for some animals: cattle, sheep/goat and horse $(\mathrm{OR}=0.63-$ $0.74, p=0.10-0.15)$. Animal care on sheep/goat was the only task significantly inversely related to lung cancer $(\mathrm{OR}=0.48$, 95\%CI: 0.25-0.94). A non-significant increased risk was associated with lifetime exposure to vineyards $(\mathrm{OR}=1.29,95 \% \mathrm{CI}$ : $0.90-1.86, \mathrm{p}=0.17)$, association strengthened among nonsmokers $(\mathrm{OR}=1.84,95 \% \mathrm{CI}: 0.99-3.44)$. An increased risk was observed for cellar work in vineyards (OR $=1.59$, 95\%CI:0.962.63, $\mathrm{p}=0.07)$, strengthened and significant in non-smokers $(\mathrm{OR}=4.06,95 \% \mathrm{CI}: 1.59-10.37)$.

Conclusion These findings support the role of a decreased lung cancer risk associated with animal-related exposures and suggest the role of hazardous exposures in vineyard, particularly for cellar work. The role of other potential hazardous exposures (including arsenic pesticides that have been used in France in vineyard until 2001 and in some fruit growing and potato production until mid 1970s) will be further investigated in this study using a crop-exposure matrix. Further efforts are needed to identify etiologic protective agents and hazardous exposures for lung cancer in farming.

\section{CANCER RISKS AMONG CANADIAN AGRICULTURAL WORKERS IN A POPULATION-BASED COHORT}

${ }^{1}$ L K Kachuri, ${ }^{2}$ Harris, ${ }^{3}$ Peters, ${ }^{3}$ Tjepkema, ${ }^{2}$ Demers. 'Occupational Cancer Research Centre, Cancer Care Ontario, Toronto, Canada; ${ }^{2}$ Occupational Cancer Research Centre, Toronto, ON, Canada; ${ }^{3}$ Statistics Canada, Ottawa, ON, Canada

\subsection{6/oemed-2013-101717.393}

Objectives Agricultural workers may be exposed to several potential carcinogens including pesticides, sensitising agents and solar radiation. Previous studies have shown increased risks of hematopoietic cancers in this population, as well as reduced risks of other types of cancer, possibly due to differences in lifestyle and risk behaviours. This study aimed to estimate cancer risks among agricultural workers in a national population-based cohort.

Methods The 1991 Canadian Census Cohort was created by Statistics Canada through probabilistic linkage between the 1991 Canadian Census (long form) to national cancer registry records from 1969-2003. Occupations were self-reported. Analyses were restricted to persons aged 74 and under who reported working at baseline (1991, total cohort $\mathrm{N}=2050300)$. Follow-up continued until December 31, 2003. Hazard ratios (HR) and corresponding 95\% confidence intervals were estimated by Cox proportional hazards modelling for all workers in agricultural occupations ( $\mathrm{N}=70300 ; 49000$ male), stratified by sex and adjusted for age at cohort entry and province of residence. 
Results There were 5437 cancer cases among agricultural workers. Among men, an increased risk of multiple myeloma was observed (HR: 1.38, 1.04-1.83), as well as oral cancer (HR: $1.28,1.09-1.51$ ), specifically lip cancer (HR: 2.94, 2.26-3.83), but had decreased risks of lung, esophageal, and liver cancers. Female agricultural workers were at increased risk of pancreatic cancer (HR: 1.44, 1.05-1.99), but decreased risk of lung, breast and cervix cancer. Higher risks of rectal cancer were also observed specifically among female farm workers and labourers (HR: 1.44, 1.02-2.04).

Conclusions Exposure to pesticides may have contributed to the increased risks of multiple myeloma in men and pancreatic cancer in women. Increased risks of lip cancer in men could be attributed to sun exposure in agricultural workers while the array of decreased risks suggests reduced smoking and alcohol consumption in agricultural workers compared to the general population.

\section{2,4-D USE AND CANCER INCIDENCE IN PESTICIDE APPLICATORS IN THE AGRICULTURAL HEALTH STUDY}

${ }^{1}$ E Beane Freeman, ${ }^{1}$ Koutros, ${ }^{1}$ Alavanja, ${ }^{1}$ Zahm, ${ }^{2}$ Sandler, ${ }^{3}$ Hines, ${ }^{4}$ Thomas, ${ }^{2}$ Hoppin, ${ }^{1}$ Blair. ${ }^{1}$ National Cancer Institute, Rockville, United States of America; ${ }^{2}$ National Institute of Environmental Health Sciences, Research Triangle Park, United States of America; ${ }^{3}$ National Institute for Occupational Safety and Health, Cincinnati, United States of America; ${ }^{4}$ Environmental Protection Agency, Research Triangle Park, United States of America

\subsection{6/oemed-2013-101717.394}

Objectives 2,4-dichlorophenoxyacetic acid (2,4-D) is one of the most widely used herbicides in the world. It has been associated with increased risk of non-Hodgkin lymphoma (NHL) in multiple epidemiologic studies, with some evidence for association with cancer at other sites. Findings from experimental studies, however, have been largely negative with respect to NHL. Within the Agricultural Health Study (AHS), a prospective cohort of licensed pesticide applicators in the United States, we evaluated use of this herbicide and multiple cancer sites.

Methods We used Poisson regression to estimate relative risks (RR) and 95\% confidence intervals for cancers that occurred from enrollment in the AHS (1993-97) through 2008. Total lifetime days of use of 2,4-D were calculated based on information provided at enrollment and at a follow-up interview conducted 5 years later. In addition, an intensity-weighting algorithm was applied to account for factors that modify exposure.

Results Overall, 78\% of the 52,324 applicators who provided information on 2,4-D use and who were cancer free at enrollment reported using 2,4-D. Among this group, there were 5,168 incident cancers. Compared to non-users, there was no association with cancer risk overall ( $\mathrm{p}$-trend $=0.68$ ), NHL overall ( $\mathrm{p}$ trend $=0.84$ ), or any sub-type of NHL with intensity-weighted lifetime days. Conversely, in the highest quartiles, there was an elevated risk of gastric cancer $(\mathrm{RR}=2.3,95 \% \mathrm{CI}: 1.1-5.2$, $\mathrm{p}$ trend $=0.03$ ) and a suggestion of elevated risk of brain cancer $(\mathrm{RR}=2.3,95 \% \mathrm{CI}: 0.9-5.7$, p-trend $=0.31)$.

Conclusions The results from this prospective study showed no association between use of 2,4-D and NHL, the cancer most often linked to this herbicide. The increased risk of gastric cancer is noteworthy but supporting data are limited. Some previous studies of brain cancer have suggested a role for pesticides, particularly herbicides; to our knowledge there is no other study specifically suggesting an association with 2,4-D.

\section{Session: 33. Hazard identification and biomarkers}

\section{SOLVENT-INDUCED DNA METHYLATION CHANGES: A TRANSLATIONAL STUDY}

${ }^{1} \mathrm{~L} G$ Godderis, ${ }^{2} \mathrm{~A} T$ Tabish, ${ }^{2} \mathrm{~K}$ P Poels, ${ }^{3} \mathrm{M}$ V Viaene, ${ }^{2} \mathrm{P} \mathrm{H}$ Hoet. ${ }^{1} \mathrm{~K} U$ Leuven/IDEWE, Leuven, Belgium; ${ }^{2} K U L$ Leuven, Occupational, Environmental and Insurance Medicine, Leuven, Belgium; ${ }^{3}$ AZ St. - Dimpna, Geel, Belgium

\subsection{6/oemed-2013-101717.395}

Objectives Workers exposed to solvents are at risk for developing cancer and neurobehavioral diseases. Evidence is growing on the role of epigenetic alterations involved in the development of both diseases. In this project, we set up a translational study to investigate the impact of solvents on DNA methylation alterations and neurobehavioral changes.

Methods First, we assessed global DNA methylation changes in human lymphoblastoid (TK6) cells in vitro in response to 10 solvents. Next, a cross sectional study was set-up to validate these results in 128 solvent workers. Liquid chromatography-mass spectrometry was used to quantify global DNA methylation profile in TK6 cells and in lymphocytes of the workers. Each participant underwent a series of tests based on the Neurobehavioral Evaluation System.

Results Benzene, hydroquinone, styrene, carbon tetrachloride and trichloroethylene induced global DNA hypomethylation in TK6 cells. DNA methylation in solvent-workers was, after correction for age, negatively associated with total exposure time $(\mathrm{r}$ $=-0.198, \mathrm{p}=0,025)$ and the cumulative exposure index $(\mathrm{r}=$ $0.244, p=0,006$ ). Age and smoking were associated with a global DNA hypomethylation, while use of alcohol was associated with hypermethylation. Interestingly, both DNA methylation and exposure were significant predictors for neurobehavioral effects in the multivariate regression models.

Conclusions We report changes in global DNA methylation as an early event in response to solvents. Global unmethylated DNA is known to dysregulate transcription, which has an impact on the gene expression and the function of cells, e.g. loss of control of cell division. These results are suggestive for the possible involvement of epigenetic mechanisms in neurodegenerative diseases and cancer. Lymphocytes are not necessarily the target tissue, but might be a good surrogate because of their accessibility and the high correlation with methylation profiles in somatic tissues.

\section{OCCUPATIONAL EXPOSURE TO IRON AMONG STEEL WORKERS INCREASED OXIDATIVE DNA DAMAGE IN PERIPHERAL LEUKOCYTES}

${ }^{1} \mathrm{M}$ Bonzini, ${ }^{2}$ Hoxha, ${ }^{2}$ Angelici, ${ }^{2}$ Bollati, ${ }^{3}$ Nordio, ${ }^{2}$ Cantone, ${ }^{2}$ Dioni, ${ }^{3}$ Baccarelli, ${ }^{4}$ Apostoli, ${ }^{2}$ Bertazzi. 'Epidemiology and Preventive medicine research Centre, University of Insubria, Varese, Italy; ${ }^{2}$ University of Milan and IRCCS Ca' Granda Maggior Hospital Foundation, Milan, Italy; ${ }^{3}$ Harvard School of Public Health, Boston, United States of America; ${ }^{4}$ Department of Experimental and Applied Medicine, University of Brescia, Brescia, Italy

\subsection{6/oemed-2013-101717.396}

Objectives The role of iron on Reactive Oxygen Species (ROS) generation by catalysing Fenton reaction have been suggested by many studies as an important factor in increasing oxidative DNA damage. Mitochondria represents an important biological source and target of ROS that induce 8-hydroxy-2'-deoxyguanosine (8-OHdG) formation. Occupational exposure to metal 